\title{
Estudio epidemiológico y clínico de los pacientes diagnosticados de tuberculosis en el área noroeste de Madrid
}

\author{
M. C. VIEIRA PASCUAL, C. BISCHOFBERGER ${ }^{1}$, C. PÉREZ DE OTEYZA² \\ Medicina de Familia. 'Medicina Preventiva. Hospital El Escorial. ${ }^{2}$ Departamento de \\ Medicina. Universidad Complutense. Madrid
} CLINICAL AND EPIDEMIOLOGICAL STUDY OF PATIENTS DIAG-
NOSED WITH TUBERCULOSIS IN THE NORTHWEST AREA OF
MADRID

\section{RESUMEN}

Objetivo: Estimar la incidencia y las características epidemiológicas y clínicas de los pacientes diagnosticado de Tuberculosis en un hospital comarcal de área noroeste de Madrid, para determinar si existe un aumento de la incidencia debido a la inmigración.

Material y métodos: Se revisaron los casos de tuberculosis desde Enero de 1996 a Junio del 2001, buscados a través del laboratorio de anatomía patológica y microbiología, recogiendo de las historias clínicas datos demográficos, clínicos y diagnósticos de la enfermedad.

Resultados: Se registraron un total de 66 tuberculosis ( 10 de ellos en inmigrantes) con una incidencia del 13/100.000 habitantes en el 1996, descendiendo hasta una incidencia del 7/100.000 en el 2000. La mediana de la edad es de 34 años con una incidencia del doble en varones $(31 / 100.000$ habitantes) respecto a las mujeres (17/100.000). El factor de riesgo predominante es el alcoholismo con 11 casos (16.7\%). Las pruebas diagnosticas más empleadas fueron la radiografía de tórax realizada en $61 / 66(92 \%)$ y fue positiva en $57(93,4 \%)$, BAAR $59(89 \%)$ siendo positiva $34(57,6 \%)$, cultivo de esputo $47(71 \%)$ dando positiva $32(68 \%)$ y el Mantoux $28(42 \%)$ siendo positiva $20(71.4 \%)$. La localización de la enfermedad es principalmente pleuro-pulmonar 50 casos $(75 \%)$. No se tipificaron prácticamente mycobacterias atípicas (5 casos). Hubo una buena respuesta al tratamiento siendo tratados con tres fármacos (isoniacida, rifampicina y piracinamida) el $88 \%$ de los pacientes con un tiempo de duración de 6,8 meses (DE: 2,8).

Conclusión: La incidencia de tuberculosis en el área noroeste de Madrid es inferior al resto de la Comunidad de Madrid, descendió en el 1997 y se ha mantenido en el 9/100.000 hasta el 2000 pese al aumento de la inmigración.

PALABRAS CLAVE: Tuberculosis. Epidemiología. Inmigración.

\section{ABSTRACT}

Objective: Estimate the incidence and the epidemiological and clini cal characteristics of patients diagnosed with tuberculosis in a county hospital located in the north western area of the Autonomous Region of Madrid, to determine if there has been an increase due to immigration.

Material and methods: Tuberculosis cases from January, 1996 to June, 2001 were analysed, researched through the Microbiology and Pathologic Anatomy Laboratory, fetching information such as demographics, clinical studies and disease diagnostics from the different medical histories.

Results: A total of 66 tuberculosis cases were registered, ten of them among immigrants, with an incidence of 13/100.000 inhabitants in 1996 , decreasing to a 7/100.000 incidence in 2000. The age average is 34 with double the incidence in males (31/100.000) than females (17/100.000). The main risk factor is alcoholism with 11 cases (16.7\%). The most com monly used diagnostic tests were the thorax radiography (61/66, 92\%) with $57(93.4 \%)$ positive identifications, culture of esputo $(47 / 66,71 \%)$ with 32 (68\%) positive ids, and the Mantoux test $(28,42 \%)$ with 20 (71.4\%) positive ids. The location of the illness is mainly pleuro-pulmo nar in 50 cases $(75 \%)$. There were almost no atypical mycobacterium found ( 5 cases). Overall, response to treatment with 3 drugs (isoniacide, rifampicine, piracinamide) was good in $88 \%$ of patients, with the avera ge treatment lasting 6.8 months (ED: 2.8).

Conclusion: The incidence of tuberculosis in the north western area of Autonomous Community of Madrid is below that of the rest of our Community. It dipped in 1997 and has been stable around 9/100.000 inhabitants until 2000 , even with the effect of immigration.

KEY WORDS: Tuberculosis. Epidemiological. Immigration.

Vieira Pascual MC, Bischofberger C, Perez de Oteyza C. Estudio epidemiológico y clínico de los pacientes diagnosticados de tuberculosis en el area Noroeste de Madrid. An Med Interna (Madrid) 2003; 20: 10-15.

\section{INTRODUCCIÓN}

La tuberculosis (TBC) es un problema de salud pública a nivel mundial que en 1993 la OMS declaró como "Emergencia Global", estimando que si no se mejoraba el control de la TBC, hasta el 2020 enfermarían unos 200 millones de personas, de los cuales fallecerían unos 70 millones .
Pese a ser una enfermedad bien conocida, está lejos de ser controlada. Ha ido en aumento en los últimos años en los países industrializados debido al impacto del VIH-SIDA (2-5) ausencia de programas de control y deterioro de la infraestructura sanitaria; aumento de la infección en sectores marginales y cambios demográficos: envejecimiento, migraciones, hacinamientos, falta de compromiso político, aumento de las resistencias.

Trabajo aceptado: 14 de octubre de 2002

Correspondencia: C. Pérez de Oteyza. Pºmilia Alarcón, 1-27. 28250 Torrelodones. Madrid. 
En 1997, un tercio de los pacientes infectados por el VIH lo estaban también por la Tuberculosis. En los países desarrollados, la TBC es la patología oportunista más frecuente en pacientes con SIDA, siendo la principal causa de muerte en pacientes infectados por $\mathrm{VIH}^{1}$.

El objetivo actual de la OMS es asegurar el diagnóstico y tratamiento adecuado para conseguir la curación de los casos de TBC activa mediante la "Estrategia DOTS" (Directly Observed Treatment Short-course) o tratamiento directamente supervisado, implicando al sistema sanitario (1).

Viendo la importancia de la enfermedad tuberculosa y su incremento decidimos realizar un estudio para estimar la incidencia y las características epidemiológicas y clínicas de los pacientes diagnosticados de TBC en El Hospital de El Escorial, zona noroeste de Madrid, y ver si existía un aumento de la incidencia debido a la inmigración ocurrida en los últimos años.

\section{MATERIAL Y MÉTODOS}

Se ha realizado un estudio retrospectivo, descriptivo, de los casos TBC diagnosticados en el Hospital comarcal de referencia del Distrito, Hospital de El Escorial. El estudio se ha realizado desde el año 1995 a Junio de 2001. En el año 1996 se estimó una población de 135.120 habitantes siendo una población joven, con máximos en los grupos de edad de 30-40 años.

Se realizó una búsqueda activa de casos de TBC a través del laboratorio de microbiología y anatomía patológica del Hospital, buscando el nombre y el número de historia clínica de las muestras positivas en BAAR o Cultivos de Löwenstein y anatomías patológicas. Una vez identificados los enfermos se procedió a la recogida de datos mediante una revisión de historias clínicas, introduciéndolos en una base de Microsoft Access 2000.

Los datos así obtenidos fueron los siguientes:

Datos demográficos: edad al inicio de los síntomas, sexo, país, tiempo de residencia, ocupación.

Datos clínicos: fecha de inicio de los síntomas, lugar de instauración del tratamiento, hospitalización, seguimiento del tratamiento, localización $1^{\circ}$ y $2^{\circ}$ de la enfermedad, factores de riesgo asociados (HIV, alcoholismo, ADVP, gastrectomizado, inmunosupresión, diabetes, silicosis, neoplasia, otros).

Datos diagnósticos: Mantoux, radiografía de tórax, ADA, BAAR y cultivo en esputo y orina, BAAR, cultivo y anatomía patológica en diversas muestras tisulares (lavado broncoalveolar, líquido pleural, biopsia pleural, biopsia ganglionar, exudado faríngeo, biopsia colon); demora en el diagnóstico, tipo de mycobacteria, si ha padecido tuberculosis previa y si ha realizado tratamiento previo.

Datos tratamiento: Fecha de inicio del tratamiento, tiempo de demora en iniciar el tratamiento, tipo de tratamiento, efectos secundarios, tiempo de seguimiento, cumplimiento y causa por la que se finalizó el tratamiento y el seguimiento, estudio de contactos.

Se realizó el estudio estadístico utilizando el programa SPSS (v 10,0).

Las variables cualitativas se describen con \%. Las variables cuantitativas con la media y desviación estándar (si cumplen con los supuestos de normalidad) o con la mediana, mínimo y máximo en caso contrario. Las frecuencias se compararon en análisis de tablas de $2 \times 2$ mediante la Chi2. La comparación de medias se hizo mediante la t de Student, en caso de normalidad de las variables o la U de Mann Whitney en caso contrario (nivel de significación $\mathrm{p}<0,05$ ).

\section{RESULTADOS}

Entre los años 1996 y junio del 2001 se diagnosticaron 82 casos de TBC en el hospital comarcal de El Escorial (Madrid), se descartaron 16 casos con BAAR positivo, por no encontrar historia, quedando 66 casos válidos de TBC para nuestro estudio.

La incidencia anual por 100.000 habitantes se recoge en la Tabla I. Como se puede observar la incidencia mayor, que corresponde a 1996, es de 18 casos (13,32/100.000 habitantes) y la menor fue en año 2000: 9 casos (6,6/100.000 habitantes).

\begin{tabular}{ccccc}
\multicolumn{6}{c}{ TABLA I } \\
INCIDENCIA ANUAL DE LA \\
\multicolumn{4}{c}{ TUBERCULO SIS PO R 100.000 HABITANTES } \\
\hline 1996 & 1997 & 1998 & 1999 & 2000 \\
\hline 13,32 & 7,40 & 9,62 & 8,88 & 6,66 \\
\hline
\end{tabular}

Como observamos en la figura 1, la distribución de la edad muestra un máximo a los 30 años con un descenso progresivo al aumentar la edad (mediana a los 34 años).

En cuanto a la distribución por sexo, 43/66 (65\%) son varones $(31,82 / 100.000)$, representando una incidencia global de casi el doble que las mujeres (17/100.000).

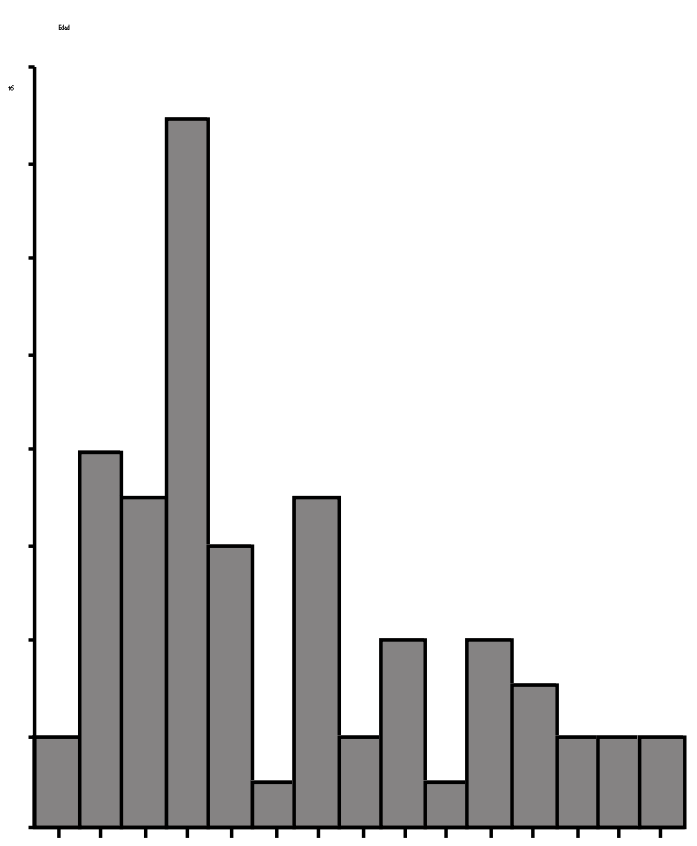

Fig. 1. Distribución de la tuberculosis según la edad en 66 casos. 
Con respecto a la procedencia de los pacientes, 10/66 (15\%) son inmigrantes, de los diez casos encontramos nueve marroquíes y un suramericano, de éstos 8/10 (89\%) son varones, no existiendo diferencias significativas respecto al sexo; el tiempo de residencia no consta en siete casos y en tres sí, siendo de 18-24 meses; la edad media de los inmigrantes (28 años) es notablemente inferior a la de los españoles (48 años), pero estadísticamente no existen diferencias significativas $(\mathrm{p}=0,11)$, esto es debido a la pequeñez muestra. Lamentablemente, no consta ocupación ni centro de trabajo en 64 de los 66 pacientes $(97 \%)$ en las historias.

El tiempo de demora en el inicio de tratamiento estuvo entre 0-396 días con una mediana de 9,50 días. El 75\% de los pacientes fueron diagnosticados antes de un mes y medio.
El tratamiento antituberculoso se inició en 64 (97\%) de los 66 casos en el hospital, de los cuales 35/66 (53\%) fueron hospitalizados y 22/66 $(13,6 \%)$ se derivaron a un hospital de crónicos donde fueron ingresados, mientras que el resto se siguió ambulatoriamente.

En la Tabla II podemos observar las pruebas para el diagnostico de la tuberculosis. La más empleada fue la radiografía de torax realizada en 61 casos $(92 \%)$ en frecuencia le sigue el BAAR 59 (89\%), cultivo de esputo 47 (71\%) y el Mantoux 28 (42\%). En cuanto a los resultados de dichas pruebas, fueron positivos: la radiografía de torax en 57 casos $(93,4 \%)$, el BAAR en $34(57,6 \%)$, el cultivo en esputo $32(68 \%)$ y el Mantoux $20(71,4 \%)$ respecto al total de los pacientes en las que fueron realizadas.

TABLA II

PRUEBAS PARA EL DIAGNÓSTICO DE LA TUBERCULOSIS EN 66 CASOS

\begin{tabular}{|c|c|c|c|c|}
\hline & \multicolumn{2}{|c|}{ Realización de prueba } & \multicolumn{2}{|c|}{ Positividad } \\
\hline & Número casos & $\%$ & $\begin{array}{l}\text { Número casos } \\
\text { positivo }\end{array}$ & Positivo \% \\
\hline Rx de tórax & 61 & 92 & 57 & 93,4 \\
\hline M antoux & 28 & 42 & 20 & 71,4 \\
\hline BAAR en esputo & 59 & 89 & 34 & 57,6 \\
\hline Cultivo en esputo & 47 & 71 & 32 & 68 \\
\hline BAAR en Iba & 10 & 15 & 3 & 30 \\
\hline Cultivo en lba & 11 & 17 & 7 & 63,6 \\
\hline Ap en lba & 9 & 14 & 2 & 22,2 \\
\hline BAAR en liq. pleural & 13 & 20 & 0 & 0 \\
\hline Cultivo en liq. pleural & 13 & 20 & 9 & 69,2 \\
\hline Ap en liq. pleural & 8 & 12 & 0 & 0 \\
\hline Ada liq. pleural & 8 & 12 & 1 & 12,5 \\
\hline BAAR en biopsia pleural & 6 & 9 & 3 & 50 \\
\hline Cultivo en biopsia pleural & 6 & 9 & 4 & 66,6 \\
\hline Ap en biopsia pleural & 9 & 14 & 3 & 33,3 \\
\hline BAAR en ganglio & 6 & 9 & 4 & 66,6 \\
\hline Cultivo en ganglio & 6 & 9 & 5 & 83,3 \\
\hline Ap en ganglio & 9 & 14 & 9 & 100 \\
\hline BAAR en orina & 3 & 4 & 1 & 33 \\
\hline Cultivo en orina & 3 & 4 & 1 & 33 \\
\hline BAAR faríngeo & 2 & 3 & 2 & 100 \\
\hline Cultivo faríngeo & 2 & 3 & 2 & 100 \\
\hline Ap faríngeo & 2 & 3 & 2 & 100 \\
\hline BAAR en colon & 1 & 1 & 1 & 100 \\
\hline Cultivo colon & 1 & 1 & 1 & 100 \\
\hline Ap colon & 1 & 1 & 1 & 100 \\
\hline
\end{tabular}


En pacientes en la figura 2 la principal localización de las lesiones tuberculosas fue la pleuro-pulmonar, con 50 pacientes $(75,8 \%)$.

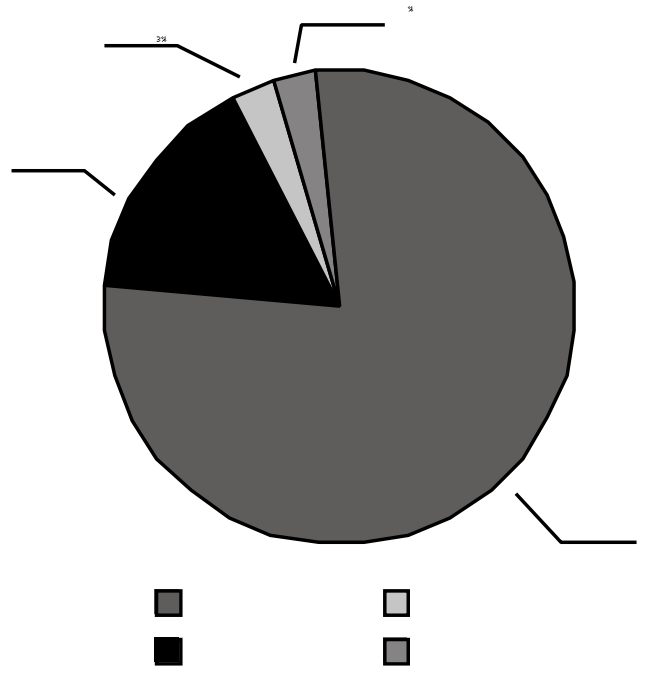

Fig. 2. Distribución de los casos de tuberculosis según su localización anatómica.

En cuanto a los antecedentes, 6 casos habían tenido tuberculosis previa y 3 habían estado en contacto con enfermo tuberculoso.

Se tipificó la bacteria en $46(70 \%)$ de los 66 casos, encontrando en 41 pacientes $(62 \%)$ el M. Tuberculoso; los 5 restantes $(8 \%)$ fueron: M. scrofulatum (1 caso), M. chalanae $(2$ casos), M fortuitum (1 caso), M.avium intracelulare (1 caso). En todos los casos de M. atípicas se encontraron resistencias a todos los antituberculosos, por lo que fueron tratados con claritromicina.

En cuanto a los factores de riesgo, que podemos observar en la figura 3, encontramos un alto porcentaje de alcohólicos (11, el 17\%), siguiéndole en frecuencia las neoplasias (9\%).

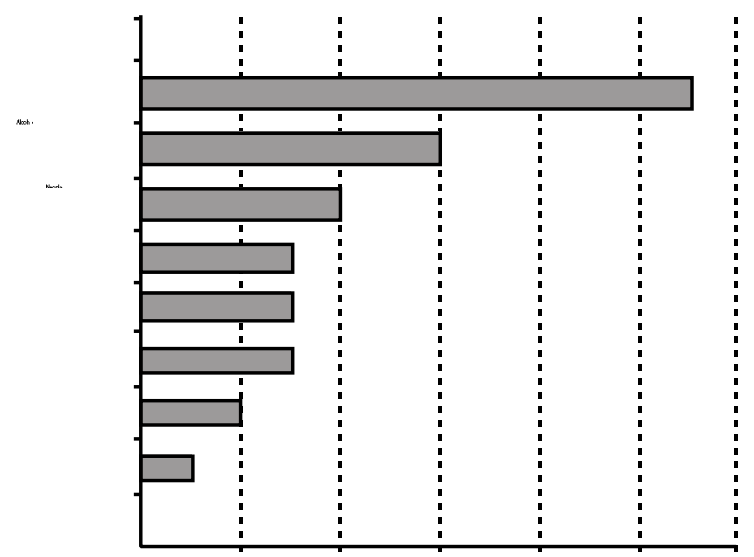

Fig. 3. Factores de riesgo en pacientes tuberculosos.
Respecto al tratamiento, 58 casos $(88 \%)$ fueron tratados con isoniacida, rifampicina y piracinamida con una duración media de 6,8 meses (DE: 2,8), por el contrario 2 (3\%) fueron tratados con isoniacida, rifampicina y etambutol con una duración de 9 meses (DE: 0) y por último 3 (4,5\%) fueron tratados con isoniacida, rifampicina, piracinamida y etambutol durante 13 meses (DE: 10,2) por ser VIH (Fig. 4).

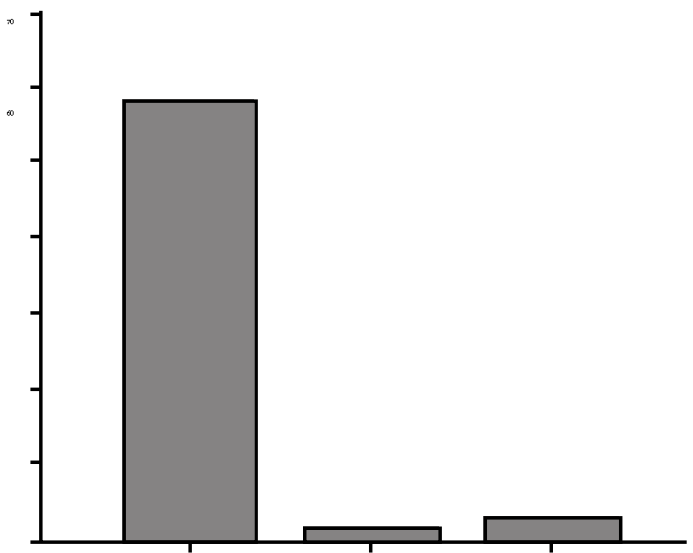

Fig. 4. Pautas de tratamiento.

Entre los diagnosticados en el hospital encontramos una pérdida de 3 casos (5\%) que fueron tratados en otro lugar por cambio de residencia. Del total de los casos en los cuales se tipificó la mycobacteria se encontraron resistencias a piracinamida en $5(8 \%)$, a rifampicina en $3(4,5 \%)$ y a isoniacida en 3 $(4,5 \%)$. Con respecto a los efectos adversos, se suspendió el tratamiento en 4 pacientes $(6 \%)$ por elevación de las transaminasas; solo hubo un caso de exantema cutáneo que no precisó suspender tratamiento, y en otro caso existía hiperuricemía previa al tratamiento, por lo que se le trató con etambutol en vez de piracinamida.

Sólo hubo dos fallecimientos entre los 66 casos. Uno de ellos por tuberculosis causada por M. atípica resistente al tratamiento. En 12 casos (18\%) hubo pérdida en el seguimiento y 6 pacientes continúan en seguimiento por no haber finalizado aún el tratamiento.

En la Tabla III se muestran las diferencias en la evolución entre inmigrantes y españoles. Llama la atención el elevado porcentaje de pérdidas para el seguimiento entre la población inmigrante $(40 \%, 4$ casos), mientras que entre los españoles tan solo fue del 14\% ( 8 casos). Dicha diferencia, aunque es clínicamente relevante no resulta estadísticamente significati$\mathrm{va}(\mathrm{p}=0,11)$,

Con respecto al estudio de contactos, lamentablemente en 62 casos (94\%) no consta en las historias si se realizó estudio y tan sólo en 4 informes $(6 \%)$ encontramos reseñados los consejos al médico de atención primaria para estudio de contactos.

Por último mencionar que 4 casos fueron diagnosticados por BAAR positivo en el mismo servicio de urgencias. 
TABLA III

DIFERENCIAS EN LA EVO LUCIÓN ENTRE INM IGRANTES Y ESPAÑO LES EN PO RCENTAJES

N Curación Traslado Exitus Pérdidas Continua

seguimiento

$\begin{array}{lcccccc}\text { Españoles } & 56 & 31(55,4 \%) & 10(17,9 \%) & 2(3,6 \%) & 8(14,3 \%) & 5(8,9 \%) \\ \text { Inmigrantes } & 10 & 3(30 \%) & 0(0 \%) & 0(0 \%) & 4(40 \%) & 2(20 \%)\end{array}$

\section{DISCUSIÓN}

La tuberculosis es una enfermedad infecciosa causada por el Mycobacterium tuberculosis, que se trasmite por gotas de 1-5 micras en forma de aerosoles contaminantes de enfermos con lesiones bacilíferas y con baciloscopia positiva. El riesgo de entrar en contacto con un caso de TBC bacilífera depende de la prevalencia de la enfermedad, el estilo de vida, el tipo de lesión del enfermo (sólida / cavitada), número de Bacilos, dinámica de las partículas y duración de la exposición.

En nuestro estudio realizado, en el área noroeste de Madrid, en el hospital comarcal de El Escorial, hemos observado que la tuberculosis tiene una incidencia anual de 9/100.000 habitantes, lo cual resulta inferior a la recogida para otras Comunidades Autónomas en el "Proyecto Multicéntrico de Investigación en Tuberculosis" (PMIT) (7). En dicho estudio se analizan 13 comunidades con una incidencia media de $38,5 / 100.000$, con un rango que oscila entre el máximo de $70,7 / 100.000$ en Galicia y un mínimo de 16,2/100.000 en Castilla la Mancha (7). Nuestra incidencia de TBC en el área de El Escorial parece mínima si la comparamos con algunas otras áreas como Santiago de Compostela, que tenía una alta incidencia: 61,9 y 68,3 por 100.000 habitantes respectivamente $(8,9)$. Merece destacar por otra parte que nuestra incidencia en el área noroeste de Madrid también es muy inferior a la del conjunto de la Comunidad de Madrid (CAM), que fue de 27,3/100.000 (10) en el año 2000. Somos conscientes, eso sí, del sesgo que puede existir al acudir los pacientes a otros hospitales de Madrid. Por otra parte, pese a que la Comunidad de Madrid tiene una incidencia de TBC mayor que el resto de Europa (15/100.000 habitantes) (11), sorprende en cambio que la zona noroeste de la CAM se encuentre por debajo de la citada media europea. En definitiva, la enfermedad en la población de la zona de El Escorial tiene una incidencia menor en relación con el resto de Europa y España, quizás por ello era refugio de tuberculosos en la primera mitad del S XX.

En nuestro estudio, durante los 5 años observados, se aprecia una disminución de la enfermedad del año 1996 al 2000. Igual ocurre en el resto de la CAM, que pasó de una incidencia de 35,30/100.000 en 1996 (12-14) hasta 27,3/100.000 habitantes en el 2000. En el resto de España durante dicho periodo se produjo también una disminución de la incidencia de la tuberculosis, a pesar de la infección por HIV, en los dos últimos años las mejoras en el tratamiento del SIDA-HIV $(3,6)$ han repercutido positivamente.

Hemos observado una incidencia de tuberculosis que es doble en hombres respecto a las mujeres, esto también ocurre en el resto de Madrid y España (7). En relación con la edad, encontramos un pico a los 30 años, lo cual difiere del estudio
PMIT que presenta dos picos, uno entre los 15-24 y otro a los 75 años. Algo parecido sucede también en la CAM.

La localización más frecuente en nuestro estudio también fue la pleuro-pulmonar, seguida de la linfática, igual que en el resto de las publicaciones $(7,8,15)$. Algunos trabajos encuentran mayor afectación de la tuberculosis extrapulmonar en los ancianos (16), algo que no hemos observado en nuestro estudio. En nuestra experiencia, el factor de riesgo más frecuente es el alcoholismo $(6,7 \%)$, a diferencia de otros estudios donde los principales factores de riesgo son la infección HIV y $\operatorname{ADVP}(7,8)$; quizás esta diferencia se pueda explicar por no existir en nuestro centro unidad de seguimiento para pacientes HIV.

Cabe destacar que nuestra demora fue inferior a 45 días en el 75\%, afortunadamente menor que la estimada en la Comunidad de Madrid en 1999 (Percentil 75: 79 días) (8).

Nos ha sorprendido encontrar sólo un $13 \%$ de inmigrantes entre nuestros tuberculosos, lo que no excede la proporción de inmigrantes en la zona noroeste de Madrid. Por ello creemos que nuestra incidencia de tuberculosis, que es baja, no depende de la inmigración, pues ésta última se ha elevado notablemente en nuestra área durante los últimos años, como ha sucedido en el resto de España (7). Por el contrario en la Comunidad de Madrid ser inmigrante es el segundo factor de riesgo (8) de padecer enfermedad tuberculosa $(17,18)$.

Pensamos que se debería mejorar la recogida de datos sobre la ocupación de los pacientes y reseñar los antecedentes de posible exposición a TBC, pues desgraciadamente ésta información no consta en las historias médicas. Igualmente cabe recordar que es obligatorio realizar un estudio de contactos en la población $(19,20)$.

En definitiva, en el área noroeste de Madrid hay una incidencia de TBC notablemente menor que en el resto de la CAM y que en los países de nuestro entorno, aunque no podemos excluir la posibilidad de un sesgo por la pérdida de pacientes HIV que acuden a otro hospital de referencia en Madrid.

Por otra parte, en cuanto al diagnóstico, las pruebas habituales de radiografía de torax, análisis en el esputo y Mantoux han dado un buen rendimiento en nuestro estudio, probablemente por la alta proporción de formas pleuro-pulmonares en nuestra serie. Cabe resaltar que en nuestra experiencia la enfermedad ha respondido muy bien al tratamiento inicial con 3 fármacos, continuando con 2 hasta los seis meses (15).

En resumen, la incidencia de la $\mathrm{TBC}$ en la zona noroeste de Madrid es inferior al resto de la comunidad, y se ha mantenido estable desde 1997 por lo que no parece haberse visto influenciada por el aumento de la inmigración experimentada en los últimos años. 


\section{Bibliografía}

1. Dirección General de Salud Públca. La tuberculosis: Un Problema de Salud Pública 1999

2. Martinez Vazquez JM, Cabarcos Ortiz de Barrón A, Barrio Gómez E. Tuverculosis e infección por VIH: epidemiologia (primera parte de las tres partes). An Med Interna (Madrid) 1997; 14: 253-6.

3. Martinez Vazquez JM, Cabarcos Ortiz de Barrón A, Barrio Gómez E. Tuverculosis e infección por VIH: patogenia (segunda de las tres partes). An Med Interna (Madrid) 1997; 14: 310-6.

4. Martinez Vazquez JM, Cabarcos Ortiz de Barrón A, Barrio Gómez E. Tuverculosis e infección por VIH: de la clínica a la prevención (tercera de las tres partes). An Med Interna (Madrid) 1997; 14: 363-8.

5. Lado Lado FL, Barrio Gómez E, Carballo Arceo E, Cabarcos Ortiz de Barrón A. Tuberculosis e infección por el Virus de la inmunodeficiencia humana. Manifestaciones clínicas y rendimiento de procedimientos diagnósticos según las distintas formas de localización de la enfermedad. An Med Interna (Madrid) 2000; 17: 27-34.

6. Del Rey Calero. Incremento actual de la tuberculosis. An Med Interna (Madrid) 1995; 12:209-11.

7. Grupo de Trabajo del PMIT. Incidencia de la tuberculosis en España: resultados del Proyecto Multicéntrico de investigación en Tuberculosis (PMIT). Med Clin (Barc) 2000; 114: 530-7.

8. Salguero Rodríguez M, Zamarrón C, Otero, et al. Estudio epidemiológico de la tuberculosis en el Área Sanitaria de Santiago de Compostela durante los años 1992,1993 y 1994. An Med Interna (Madrid) 1996; 13: 111-4.

9. Salguero Rodríguez M, Zamarrón C, Gónzalez Barcala J, et al. Estudio epidemiológico de la tuberculosis en el Área Sanitaria de Santiago de Compostela durante los años 1995,1996.1997 y 1998. An Med Interna (Madrid) 2001; 18: 20-3.
10. Informe regional de casos de TBC de la Comunidad de Madrid. Informe del año 1999. Boletín epidemiológico de la Comunidad de Madrid 2000; 7(2): 3-45

11. EuroTB and the National Coordinators for Tuberculosis Surveillance in the Who European Region. Surveillance of Tuberculosis in Europa: report on Tuberculosis cases notified in 19997. Sr Maurice, France: Eurotb, $1999<$ http://www.cese.org/eurotb<

12. Comunidad de Madrid. Informe del Registro Regional de casos de TBC 1996. Boletín epidemiológico de la Comunidad de Madrid 1998; 5 (12): 3-28.

13. Comunidad de Madrid. Informe del Registro Regional de casos de TBC 1997. Boletín epidemiológico de la Comunidad de Madrid 1998; 5 (21): 4-33.

14. Comunidad de Madrid. Informe del Registro Regional de casos de TBC 1998. Boletín epidemiológico de la Comunidad de Madrid 2000; 6 (8): 3-33.

15. Lado Lado FL, Tuñez Bastida V, Golpe Gómez AL. Tuberculosis extrapulmonar en nuestro medio. Formas de presentación. An Med Interna (Madrid) 2000; 12: 637-41.

16. Lado Lado F:L, Tuñaz Bastida V, Golpe Gómez A.L, Cabarcos Ortiz De Barrón M.L. Perez Del Molino M.L. Tuberculosis en pacientes ancianos. Formas de presentación. An Med Interna (Madrid) 2002; 19 (3): 111-4.

17. Ruiz Manzano J; Tuberculosis e inmigrantes. Med Clin (Barc) 2000; 114: 257-8.

18. De Paula F, Rivas Clemente J, Nácher Canches M, Corrillero Martín J, García-Herreros Madieño T. Prevalencia de la tuberculosa entre los inmigrantes magrebíes. Med Clin (Barc) 2000; 114: 245-9.

19. Sánchez Moreno A, Villena L, Castro JM, Aguayo S, Pujol E. Estudio de tuberculosis en la prisión de Huelva. Am J Med 1984; 84: 833-5.

20. Extrenera Montero F, Moyano Axost R, Gómez Pozo B. Exposure to Mycobacterium tuberculosis during a bus travel.Med Clin (Barc) 2001; 116 (5): 182-5. 